\title{
Comportamento reológico de néctar de cagaita descrito pelo modelo lei de potência
}

\author{
Fernando Luiz de Oliveira ${ }^{1}$, Camila Fernanda Dias de Oliveira² ${ }^{2}$ Ellen Godinho Pinto \\ ${ }^{1}$ Instituto Federal Goiano-IF Goiano, Campus de Morrinhos, Morrinhos, Goiás, Brasil. E-mail: ferdinandomf@yahoo.com.br, \\ ellen.godinho@ifgoiano.edu.br. \\ ${ }^{2}$ Universidade Federal de Goiás-UFG, Campus Samambaia, Samambaia, Goiás, Brasil. E-mail: camilaferdias @ gmail.com.
}

Recebido: 15/04/2016; Aceito: 07/07/2016.

\section{RESUMO}

Neste trabalho, foi determinado o comportamento reológico do néctar de cagaita sob a pasteurização lenta e rápida mediante um reômetro, nos intervalos de temperatura de 5,15 e $25^{\circ} \mathrm{C}$. Foi realizado o ajuste pelo modelo Lei de Potência, o modelo se demonstrou adequado para descrever o comportamento reológico do néctar de cagaita. O néctar de cagaita sob os dois tipos de pasteurização demonstrou comportamento não newtoniano e demostrando característica de fluido pseudoplástico. Os índices de comportamento de consistência decresceram com o aumento da temperatura.

Palavras-chave: Pasteurização lenta, pasteurização rápida, viscosidade, taxa de cisalhamento.

\section{Rheological behavior of cagaita nectar described by the power law model}

\begin{abstract}
In this study, the rheological behavior of cagaita nectar under fast and slow pasteurization by a rheometer in the temperature intervals of 5,15 and $25{ }^{\circ} \mathrm{C}$ were determined. The data were adjusted by the Power Law model. The results indicated that the model proved adequate to describe the rheological behavior of cagaita nectar. The nectar cagaita under the two types of pasteurization showed non-Newtonian behavior but pseudoplastic fluid. The consistency behavior index decreased with increasing temperature.
\end{abstract}

Key words: slow pasteurization; rapid pasteurization; viscosity; Shear rate. 


\section{Introdução}

Entre as espécies frutíferas nativas da região do cerrado, destaca-se a cagaita (Eugenia dysenterica DC.). $\mathrm{O}$ fruto de cagaita possui formato globoso, levemente achatado, casca frágil de coloração amarelo-claro e polpa com sabor agradável, levemente ácida. A exploração tecnológica da cagaita mediante desenvolvimento de produtos alimentares pode representar alternativa de significância econômica, social e nutricional para as famílias do cerrado brasileiro (BEDETTI et al., 2013).

Deve-se considerar a sua utilização na elaboração de novos produtos, afim de atender expectativas de consumidores, quanto a novidades no setor alimentício, além de se constituir em mais uma opção de renda de pequenos produtores no cerrado.

A cagaita pode ser utilizada na fabricação de diversos produtos. No entanto, devido às suas características químicas, como pH ácido, alta acidez titulável e elevada umidade (CARDOSO et al., 2011), o fruto mostra-se apto ao desenvolvimento de bebidas, especialmente o néctar. O termo néctar é usado para bebida não fermentada, destinada ao consumo direto, obtida a partir da diluição em água potável da parte comestível da fruta, ou de seu extrato, e de açúcares (BRASIL, 2009).

As indústrias de sucos de frutas têm a preocupação de melhorar e automatizar a produção dos seus produtos. Nos sucos e néctares durante o processo, podem apresentar variações em suas concentrações e temperaturas. Estes são submetidos a várias operações unitárias, tais como bombeamento, evaporação, secagem e pulverização. Para um correto dimensionamento, o conhecimento dos parâmetros reológicos desses produtos é de fundamental importância (SILVA et al., 2008).

O comportamento reológico é considerado uma ferramenta analítica, a qual fornece uma melhor compreensão da organização estrutural dos alimentos. Vários fatores afetam o comportamento reológico das polpas de frutas, destacando-se entre estes, a temperatura, sólidos solúveis e o tamanho das partículas (GONÇALVES et al., 2013).

O comportamento reológico do néctar pode ser descrito por diversos modelos empíricos, que relacionam tensão de cisalhamento e taxa de deformação (SILVA et al., 2012).

Poucos estudos ainda foram realizados sobre o néctar de cagaita e sua industrialização. Dessa maneira, o objetivo deste trabalho foi realizar uma caracterização reológica de modo a contribuir para o dimensionamento na indústria do néctar de cagaita sob a pasteurização lenta e rápida.

\section{Material e Métodos}

Os frutos da cagaitas foram obtidos no município de Guarda- Mor, Minas Gerais, posteriormente foram levados ao Laboratório de Agroindústria do Instituto Federal Goiano- Campus Morrinhos. A polpa da cagaita foi extraída manualmente e congeladas a $-18^{\circ} \mathrm{C}$, para posterior análises.

Para a formulação do néctar, foram utilizadas $37,5 \%$ de polpa de cagaita; $52,5 \%$ de água mineral e $10 \%$ de sacarose comercial e homogeneizou-se em processador doméstico. Após o preparo, o néctar foi armazenado em garrafas de plásticos de polietileno para posterior pasteurização.

O néctar formulado foi submetido as pasteurização aqui estudada neste trabalho: lenta $\left(65{ }^{\circ} \mathrm{C} / 30 \mathrm{~min}\right)$ e rápida $\left(72-75{ }^{\circ} \mathrm{C} / 15 \mathrm{~s}\right)$, a pasteurização foi realizada em banho-maria e resfriamento em banho com gelo.

As propriedades reológicas foram determinadas segundo Vidal et al. (2004), utilizando-se reômetro (Physica, MCR 101, Ostfildern, Germany), foram realizadas no laboratório Lab Multi, na UFG. As medidas foram realizadas nas temperaturas de 5, 10, 25 ${ }^{\circ} \mathrm{C}$, por incluírem a temperatura típica de prateleira do produto acabado $\left(10^{\circ} \mathrm{C}\right)$, a temperatura representativa de resfriamento nas indústrias $\left(5^{\circ} \mathrm{C}\right)$ e a temperatura ambiente $\left(25^{\circ} \mathrm{C}\right)$.(FERREIRA et al., 2008).

$\mathrm{O}$ procedimento de medida para todas as amostras foi realizado em triplicata, sendo obtidos então valores de tensão de cisalhamento relativos à taxa de deformação aplicada, que variaram na faixa de 0 a 500 $\mathrm{s}^{-1}$, e o valor final dos parâmetros foi a média resultante das três leituras.

O modelo Lei da Potência (Equação 1) foi ajustado ao dados obtidos de tensão de cisalhamento versus taxa de deformação.

$$
\tau=\mathrm{K} \Upsilon \mathrm{n}
$$

$$
\begin{aligned}
& \tau: \text { Tensão de cisalhamento }(\mathrm{Pa}) ; \\
& \Upsilon: \text { Taxa de deformação }(\mathrm{s}-1) ; \\
& \mathrm{K} \text { : Índice de consistência (Pa1/2.sn); } \\
& \mathrm{n} \text { : Índice de comportamento (adimensional). }
\end{aligned}
$$

\section{Resultados e Discussão}

As Figuras 1 e 2 mostram os efeitos da temperatura no comportamento reológico no néctar de cagaita nas temperaturas de pasteurização $65^{\circ} \mathrm{C}$ e $75^{\circ} \mathrm{C}$, respectivamente; são apresentados os pontos experimentais médios e suas respectivas curvas de ajuste do modelo Lei de Potência.

Observa-se, para taxa deformação usada, a tensão de cisalhamento diminui com o aumento da temperatura. De acordo com Bezerra et al. (2013), com aumento da temperatura, observa-se a inclinação das curvas diminui e taxa de deformação aumentar. Isto evidencia uma 
diminuição da viscosidade aparente com o aumento da taxa de deformação. Portanto, pode-se notar que no néctar pasteurizado rápido as temperaturas tiveram uma influência pequena, o que diferenciou da pasteurização lenta que teve uma notória influência das temperaturas nas inclinações das curvas de deformação.

Os parâmetros relativos ao modelo Lei de Potência obtidos por meio de ajustes experimentais se encontra na Tabela 1. O modelo Lei de Potência adequou-se para descrever o comportamento reológico do néctar de cagaita tanto para a pasteurização lenta $\left(65^{\circ} \mathrm{C}\right)$ como na pasteurização rápida $\left(75^{\circ}\right)$ nas temperaturas neste trabalho estudadas, apresentando valores de coeficiente de determinação (r), superiores 0,92 , portanto pode-se observar que o modelo teve uma melhor adequação para o néctar pasteurizado rápido. Verifica-se que o índice de comportamento (n) em todas as temperaturas estudadas apresentou valores inferiores a um (1), indicando um fluido pseudoplástico para o néctar de cagaita. Comportamento semelhante foram observados por Carneiro et al. (2013), em suco de uva e por Silva et al. (2012), em bebidas mistas de cajá e manga.

Com o aumento da temperatura, analisou-se uma diminuição no índice de comportamento (n) em ambas pasteurizações, em todas faixas de temperaturas analisadas $\left(5,10\right.$ e $\left.25^{\circ} \mathrm{C}\right)$, com decréscimo de $69,59 \%$ para o néctar de cagaita pasteurizado lentamente e $68,97 \%$ para o néctar de cagaita pasteurizado rápido. Esse comportamento também foi observado por Bezerra et al. (2013), no suco misto de frutas tropicais.

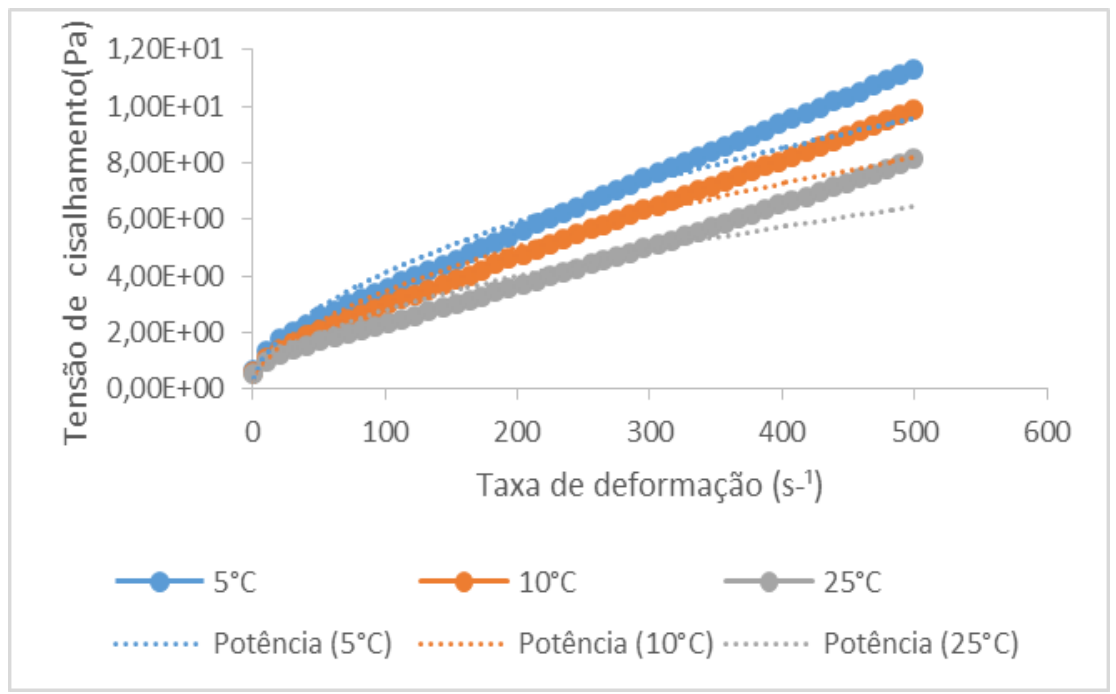

Figura 1. Tensão de cisalhamento versus taxa de cisalhamento a $65^{\circ} \mathrm{C}$.

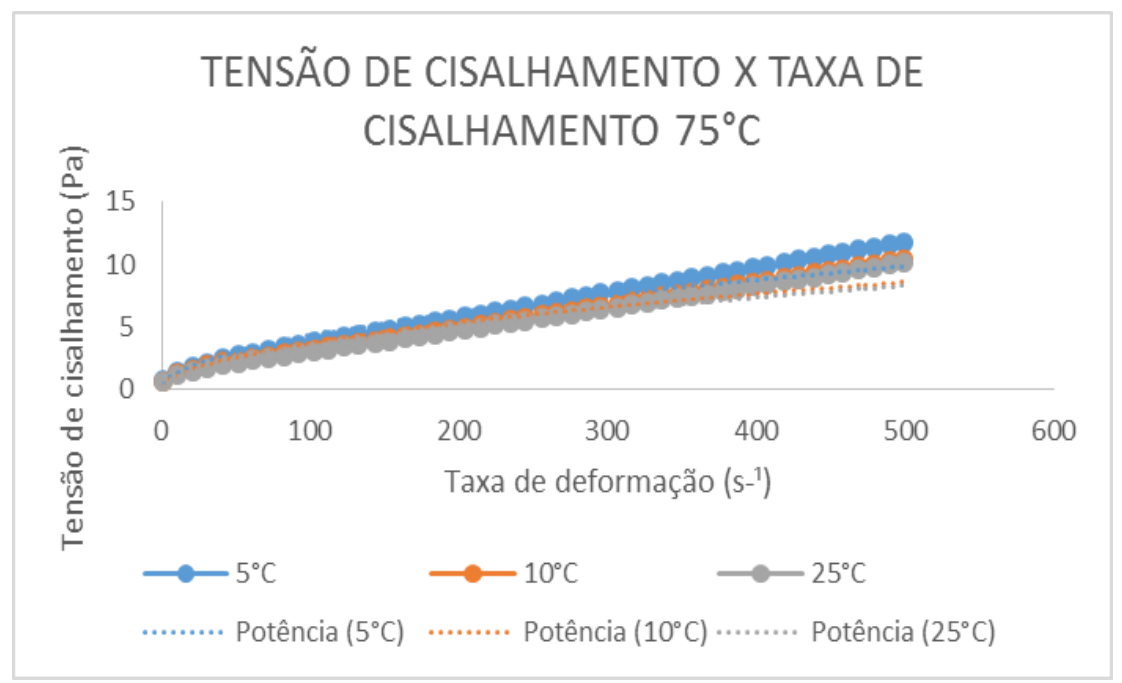

Figura 2. Tensão de cisalhamento versus taxa de cisalhamento a $75{ }^{\circ} \mathrm{C}$. 
Tabela 1. Parâmetros reológicos obtidos pelo modelo Lei de Potência do néctar de cagaita em diferentes temperaturas de pasteurização.

\begin{tabular}{ccccc}
\hline Amostra & Parâmetros & \multicolumn{3}{c}{ Temperatura } \\
\cline { 3 - 5 } & & $5^{\circ} \mathrm{C}$ & $10^{\circ} \mathrm{C}$ & $25^{\circ} \mathrm{C}$ \\
\hline $65{ }^{\circ} \mathrm{C}$ & $\mathrm{K}$ & 0,5250 & 0,5339 & 0,5195 \\
& $\mathrm{n}$ & 0,3673 & 0,297 & 0,2556 \\
& $\mathrm{r}$ & 0,9515 & 0,9433 & 0,9243 \\
$75^{\circ} \mathrm{C}$ & $\mathrm{K}$ & 0,5138 & 0,5241 & 0,5467 \\
& $\mathrm{n}$ & 0,4042 & 0,3306 & 0,2788 \\
& $\mathrm{r}$ & 0,9446 & 0,9415 & 0,9438 \\
\hline
\end{tabular}

$\mathrm{Na}$ Figura 3 e 4, apresentam-se as curvas da viscosidade aparente $(\eta)$ do néctar de cagaita na pasteurização lenta e rápida, demostrando a relação entre viscosidade aparente e taxa de deformação. Verifica-se o decréscimo da viscosidade aparente, considerando-se isoladamente os aumentos de temperatura e taxa de deformação, como esperado.

Observa-se, na Figura 3 e 4, comportamento similares, formando duas regiões distintas, às quais correspondem comportamentos estruturais bem diferenciados. A primeira região, zona de taxa de deformação inferior a $100 \mathrm{~s}^{-1}$, na qual as forças de agregação começam a ser superadas pelas forças de cisalhamento conduzindo o alinhamento das partículas, de modo que a viscosidade aparente do néctar decresce exponencialmente. Na segunda região, zona de taxa de deformação superior a $100 \mathrm{~s}^{-1}$, o estado de alinhamento e orientação das partículas encontra-se totalmente estabelecido, e a viscosidade aparente do néctar tende a ficar constante, indicando um comportamento newtoniano para o néctar.

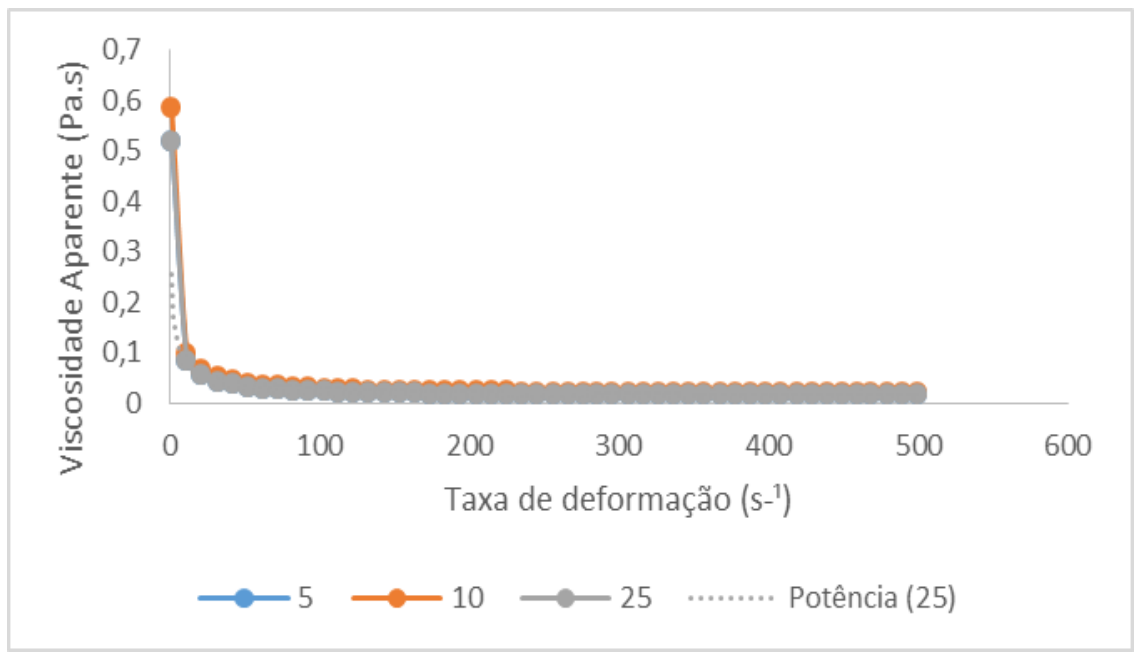

Figura 3. Curva da viscosidade aparente $(\eta)$ do néctar de cagaita pasteurização lenta $\left(65^{\circ} \mathrm{C}\right)$.

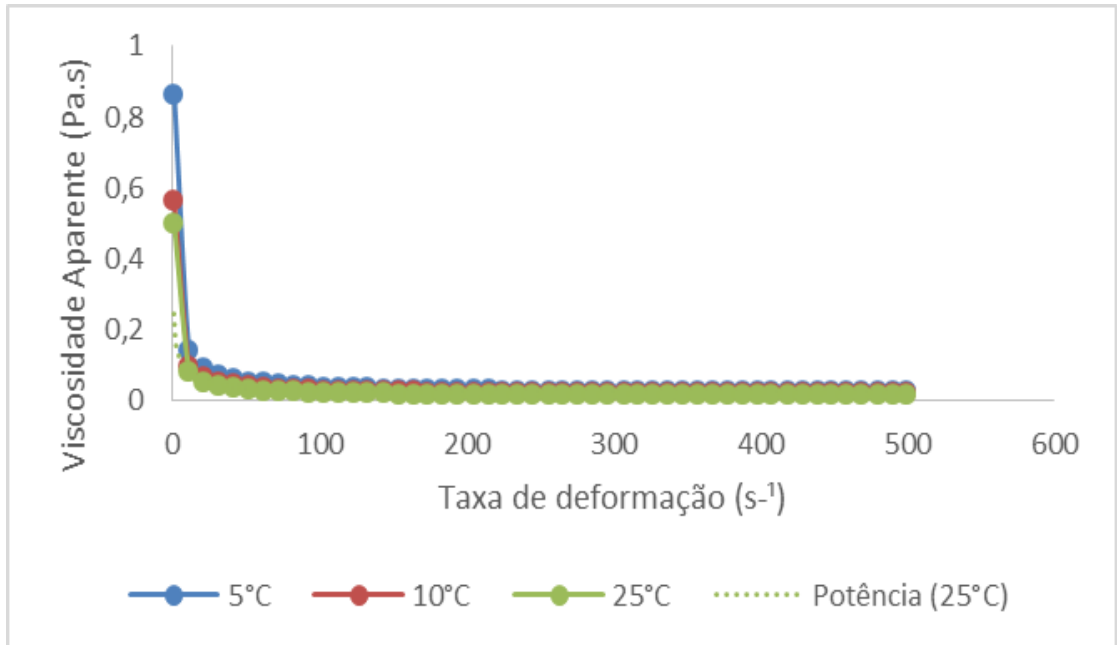

Figura 4. Curva da viscosidade aparente $(\eta)$ do néctar de cagaita pasteurização rápida $\left(75^{\circ} \mathrm{C}\right)$. 


\section{Conclusões}

As pasteurizações lenta e rápidas não tiveram influencias significativas sobre o comportamento reológico do néctar de cagaita.

Podendo concluir que o modelo Lei de Potência forneceu bons ajustes nas temperaturas estudadas, caracterizando o néctar de cagaita como um fluido pseudoplástico. Com o aumento da temperatura observou-se um aumento no índice de comportamento (n), nas pasteurizações estudadas. Podendo o néctar de cagaita em ambas as pasteurizações serem industrializadas, para a comercialização.

\section{Referências Bibliográficas}

BEDETTI, S. F.; CARDOSO, L. M.; SANTOS, P. R. G.; DANTAS, M. I. S.; PINHEIRO-SANT'ANA, H. M. Néctar de cagaita (Eugenia dysenterica dc.): desenvolvimento, caracterização microbiológica, sensorial, química e estudo da estabilidade. B.CEPPA, Curitiba-PR, v. 31, n. 1, p. 125-138, 2013. http://dx.doi.org/10.5380/cep.v31i1.32709

BEZERRA, C. V.; SILVA, L. H. M.; COSTA, R. D. S.; MATTIETTO, R. A.; RODRIGUES, A. M. C. Comportamento reológico de suco misto elaborado com frutas tropicais. Brazilian Journal of Food Techonology, Campinas-SP, v. 16, n. 2, p. 155-162, 2013.

BRASIL. Ministério da Agricultura. Decreto ${ }^{\circ}$ 6871, de 4 de junho de 2009. Dispõe sobre a padronização, a classificação, o registro, a inspeção e a fiscalização da produção e do comércio de bebidas. Diário Oficial [da República Federativa do Brasil], Brasília-DF, 04 de junho de 2009.

CARDOSO, L. M.; MARTINO, H. S. D.; MOREIRA, A. V. B.; RIBEIRO, S. M. R.; PINHEIRO-SANT'ANA, H. M. Cagaita (Eugenia dysenterica DC.) of the Cerrado of Minas Gerais, Brazil: Physical and chemical characterization, carotenoids and vitamins. Food Research International, Viçosa-MG, v. 44, p. 2151-2154, 2011. http://dx.doi.org/10.1016/j.foodres.2011.03.005
CARNEIRO, A. P. G.; ABREU, D. A.; SOARES, D. J.; COSTA, E. A.; SILVA, L. M. R.; BARBOSA, L. C.; SOUZA, P. H. M.; FIGUEIREDO, R. W. Avaliação da rotulagem, caracterização química, físico-química e reológica de Néctares de uva comercializados na cidade de Fortaleza - CE. Alimentos e Nutrição, Araraquara-SP, v. 24, n. 2, p. 241-249, 2013

FERREIRA, G. M.; GUIMARÃES, M. J. O. C.; MAIA, M. C. A. Efeito da temperatura e taxa de cisalhamento nas propriedades de escoamento da polpa de cupuaçu (Theobroma grandiflorum Schum) integral. Revista Brasileira Fruticultura, Jaboticabal-SP, v. 30, n. 2, p. 385-389, 2008.

GONÇALVES, M. V. A.; SILVA, J. P. L.; MATHIAS, S. P.; ROSENTHAL, A.; CALADO, V. M. A. Caracterização físicoquímica e reológicas da polpa de Cupuaçu congelada (Theobroma grandiflorum schum). Perspectivas online, Campos dos Goytacazes-RJ, v. 3, n. 7, p. 56-53, 2013.

SILVA, L. M. R.; MAIA, G. A.; SOUZA, P. H. M.; RAMOS, A. M.; AFONSO, M. R. A.; FIGUEIREDO, R.W. Comportamento reológico de néctares mistos de caju, manga e acerola descritos pelo Modelo de Casson. Brazilian Journal of Food Techonology, Campinas-SP, v.15, n. 3, p. 237-242, 2012.

SILVA, M. R.; LACERDA, D. B. C. L.; SANTOS, G. G.; MARTINS, D. M, O. Caracterização química de frutos nativos do cerrado. Ciência Rural, Santa Maria-RS, v.38, n.6, p.1790-1793, 2008. http://dx.doi.org/10.1590/S010384782008000600051

VIDAL, J. R. M. B.; PELEGRINE, D. H.; GASPARETTO, C. A. Efeito da temperatura no comportamento reológico da polpa de manga (Mangífera indica L-Keitt). Ciência e Tecnologia de Alimentos, Campinas-SP, v. 24, n. 1, p. 39-42, 2004. 